\title{
Inhibition of Haemoglobin Glycosylation, Glucose Uptake and In Vitro Antidiabetic Activities of Solvent Fractions from Daucus Carota Seed
}

\author{
Habibu Tijjani* and Sadiq Adamu Imam
}

Department of Biochemistry, Bauchi State University, Gadau 751105, Nigeria

Received $20^{\text {th }}$ February, 2021, Accepted $2^{\text {nd }}$ May, 2021

DOI: 10.2478/ast-2021-0004

*Corresponding author

Habibu Tijjani E-mail: haatscific@gmail.com

Tel: $+234-8037327138$

\begin{abstract}
Diabetes mellitus (DM) is a metabolic disorder which result from either absolute or relative insulin deficiency and may lead to dysfunction in some organ systems. Pancreatic $\alpha$-glucosidase and $\alpha$ - amylase inhibition are an effective strategy to decrease levels of postprandial hyperglycemia through starch control breakdown. The aim of the study is to assess the in vitro antidiabetic activities of fractions from Daucus carota seed extract, a plant used traditionally for the treatment of diabetes mellitus. The aqueous extract (AQE) of Daucus carota seed was partitioned in ethyl acetate (EAE), $n$ - hexane (HEX) and diethyl ether (DEE) to obtain the various fractions. The AQE and EAE expressed significant $\alpha$ - amylase inhibitory activity with IC $_{50}$ values of $637.0 \pm 18.6 \mu \mathrm{g} / \mathrm{mL}$ and $603.0 \pm 25.8 \mu \mathrm{g} / \mathrm{mL}$ respectively. AQE, EAE, HEX and DEE expressed $\alpha$ - glucosidase inhibitory activity with $\mathrm{IC}_{50}$ value of $135.85 \pm 1.21,147.59 \pm 0.57$, $132.64 \pm 1.17$, and $143.56 \pm 0.49 \mu \mathrm{g} / \mathrm{mL}$ respective compared with acarbose (ACA) with $5.42 \pm 0.20 \mu \mathrm{g} / \mathrm{mL}$. Furthermore, DEE fraction expressed inhibitory effects on \% glucose uptake in yeast cell comparable with metronidazole. All Daucus carota fractions expressed various inhibitory effects on haemoglobin glycosylation at a concentration of $200-1000 \mu \mathrm{g} / \mathrm{mL}$. The results show that fractions from the aqueous seed extract of Daucus carota possess in vitro antidiabetic potentials with EAE and HEX fractions having most promising inhibitory activities against $\alpha$-amylase and $\alpha$-glucosidase respectively.
\end{abstract}

Keywords: Daucus carota; seed; in vitro; antidiabetic activities; glucose uptake; haemoglobin glycosylation 


\subsection{Introduction}

Diabetes mellitus (DM) is a metabolic disorder with disturbances in carbohydrate, fat and protein metabolism characterized by a chronic hyperglycemic conditions. DM may result from either absolute or relative insulin deficiency and may lead to dysfunction in some organ systems (WHO, 2009). DM has shown a tremendous increase in occurrence through their transition from epidemiology studies in a recent study (Uloko et al., 2018). DM has also pose real challenges to the health sector through finances and budgeting (Uloko et al., 2018). Recent prevalence figures published by International Diabetes Federation (IDF) indicated about 463 million adults are living with DM worldwide, with 1 in 2 (about 232 million) living with diabetes undiagnosed and about 4.2 million deaths (International Diabetes Federation, 2019). Due to the developing economy of Africa and Asia, their economy contributes a significant fraction of the observed prevalence figures. More so, there are concerns arising from the complications of DM alongside the observed increased prevalence of the disease (Uloko et al., 2008). Effective management and treatment of DM are therefore of immense importance and area of research.

Daucus carota L. (Carrot) is an important crop from the family Apiaceae with worldwide distribution. Carotenoids and anthocyanins are among the major antioxidant pigments found in carrots (Olatunde et al., 2020). Traditionally, D. carota seed are used to treat a spectrum of diseases, including DM which is associated with oxidative stress and hyperlipidaemia (Khaki, 2011; Pouraboli and Ranjbar, 2015, Tijjani et al., 2020a; 2020b). Furthermore, the seeds have analgesic (Vasudevan et al., 2010), anti-inflammatory (Vasudevan et al., 2010), hepatoprotective (Singh et al., 2012), in vitro and in vivo antioxidant activities (Tijjani et al., 2020a; 2020b; 2020c). Medicinal plant is moving from a simple to diverse used with a large number of researches reporting the benefits of whole plants and specific components in them for medicinal usage. Their components haven't reported side effects, which have been earlier, reported by synthetic drugs, making traditional plant based treatments preferred choice for treatment of diseases (Tijjani et al., 2017). The present studies evaluated the in vitro antidiabetic activities of the various fractions of Daucus carota seed.

\subsection{Experimental}

\section{Plant Material}

Daucu scarota L. seeds were purchased from AlheriManoma, Musty Agroallied Nigeria, LTD and were identified by Mr Azila Joseph, a curator with the Federal College of Forestry, Jos, Plateau state and a voucher specimen with the number FHJ 288 was deposited.

\section{Chemicals and Reagents}

Sucrase, $\alpha$ - amylase, $\alpha$ - glucosidase and 3, 5-dinitrosalicylic acid (DNSA) were purchased from Sigma-Aldrich, Germany. Glucose kit was purchased from Randox Laboratory. All other reagents used were of analytical grade.

\section{Preparation of Aqueous Carrot Seed Extract}

Daucus carota L. seed was pulverized into powder and $190 \mathrm{~g}$ of the powder was dissolve in $1900 \mathrm{~mL}$ of distilled water for 24 hours. The aqueous extract (AQE) was filtered using Whatman filter paper (No 1). The filtrate was concentrated at $40^{\circ} \mathrm{C}$ using a rotary evaporator.

\section{Partitioning of Crude Aqueous Extract}

The aqueous extract was partitioned in water $(100 \mathrm{~mL})$ and ethyl acetate (EAE, $200 \mathrm{~mL}$ ) in a separation funnel. The various partitioned fractions (EAE and Aqueous EAE) was concentrated at $40^{\circ} \mathrm{C}$ using a rotary evaporator to obtain the ethyl acetate (EAE) and aqueous extract. The procedure was repeated for $\mathrm{n}$ - hexane (HEX) and diethyl ether (DEE) by partitioning the aqueous fractions obtained from each step of extraction.

\section{$\alpha$-amylase Inhibition Assay}

A $500 \mu \mathrm{L}$ of AQE, EAE, HEX, DEE and acarbose (ACA) at $100-1000$ $\mu \mathrm{g} / \mathrm{mL}$ was added to $500 \mu \mathrm{L}$ of phosphate buffer $(0.02 \mathrm{M}, \mathrm{pH} 6.9)$ containing sodium chloride $(6 \mathrm{mM})$ and $\alpha$-amylase $(0.5 \mathrm{mg} / \mathrm{mL} ; 0.04$ units Porcine Pancreatic $\alpha$-amylase (PPA)) solution and incubated for $10 \mathrm{~min}$ at $37^{\circ} \mathrm{C}$. After which, $500 \mu \mathrm{L}$ of starch solution (0.5\%) in sodium phosphate buffer $(0.02 \mathrm{M}, \mathrm{pH}$ 6.9) was added to each tube and incubated for $10 \mathrm{~min}$ at $37^{\circ} \mathrm{C}$. The reaction was stopped by adding 1 $\mathrm{mL}$ of 3, 5-dinitrosalicylic acid (DNSA) colour reagent. The reaction mixture was incubated in a boiling water bath for $5 \mathrm{~min}$, cold to room temperature and then diluted with $10 \mathrm{~mL}$ distilled water. The absorbance was measured at $540 \mathrm{~nm}$. Control represent $100 \%$ enzyme activity and was conducted by replacing fraction and standard with vehicle (Kuppusamy et al., 2011).

\section{$\alpha$-glucosidase Inhibition Assay}

$\alpha$-glucosidase inhibitory assay was determined by incubating $1 \mathrm{~mL}$ solution of starch substrate $(2 \% \mathrm{w} / \mathrm{v})$ with $1 \mathrm{~mL}$ Tris buffer $(0.2 \mathrm{M}, \mathrm{pH}$ 8.0) and various concentrations of AQE, EAE, HEX, DEE and acarbose (ACA) at $37^{\circ} \mathrm{C}$ for $5 \mathrm{~min}$. The reaction was initiated by adding $1 \mathrm{~mL}$ of $\alpha$-glucosidase enzyme $(1 \mathrm{U} / \mathrm{mL})$ to it followed by incubation at $37^{\circ} \mathrm{C}$ for $10 \mathrm{~min}$. The reaction was stop by heating the reaction mixture for 2 min in boiling water bath. The amount of glucose liberated was measured using glucose oxidase peroxide method (Andrade-Cetto et al., 2008; Kuppusamy et al., 2011).

\section{Determination of Glucose Uptake in Yeast Cells}

Commercial baker's yeast was dissolve in distilled water and subjected to centrifuge repeated at $3000 \mathrm{~g}$ for $5 \mathrm{~min}$, until a clear supernatant fluids is obtained. From the suspension mixture a $10 \%(\mathrm{v} / \mathrm{v})$ was prepared in distilled water. AQE, EAE, HEX, DEE and metronidazole $(200-1000 \mu \mathrm{g} / \mathrm{mL})$ were pipetted to $1 \mathrm{~mL}$ of glucose solution $(5,10,25$ $\mathrm{mM}$ ) and incubated for $10 \mathrm{~min}$ at $37^{\circ} \mathrm{C}$. Reaction was initiated by adding $100 \mu \mathrm{L}$ of the prepared $10 \%$ yeast suspension followed by vortexing and incubation at $37^{\circ} \mathrm{C}$ for $60 \mathrm{~min}$. The preparation were centrifuged at $2500 \mathrm{~g}$ for $5 \mathrm{~min}$ and amount of glucose estimated in the supernatant using glucose oxidase method (Cirillo, 1962). Metronidazole was used as reference drug. 


\section{Inhibition of Haemoglobin Glycosylation}

To $1 \mathrm{~mL}$ of haemoglobin solution, $25 \mu \mathrm{L}$ of gentamycin and $25 \mu \mathrm{L}$ of AQE, EAE, HEX, DEE and gentamycin was pipetted into a test tube. The reaction was initiated by the addition of $1 \mathrm{~mL}$ of $2 \%$ glucose in phosphate buffer $(0.01 \mathrm{M}, \mathrm{pH} 7.4)$ and incubate at room temperature in the dark. The concentrations of glycated haemoglobin at the incubation period of 0,24 and 72 hours was estimated spectrophotometrically at 443nm (Adisa et al., 2004).

\section{Data Analysis}

Data are presented as mean \pm standard error of mean (SEM) following Duncan multiple range test using SPSS version 20, SPSS Inc., Chicago. IL, USA. The significant values were determined at $\mathrm{p}<0.05$.

\subsection{Results and Discussion}

The fractions obtained express significant in vitro antidiabetic properties. AQE and EAE expressed $\alpha$ - amylase activities with $\mathrm{IC}_{50}$ values of $637.0 \pm 18.6 \mu \mathrm{g} / \mathrm{mL}$ and $603.0 \pm 25.8 \mu \mathrm{g} / \mathrm{mL}$ (Table 1). However, HEX, and DEE expressed $\alpha$ - amylase inhibitory activities greater than $1000 \mu \mathrm{g} / \mathrm{mL}$ compared with acarbose with $\mathrm{IC}_{50}$ values of $7.2 \pm 0.8 \mu \mathrm{g} / \mathrm{mL}$. The $\mathrm{IC}_{50}$ values for $\alpha$ - glucosidase of the various fraction ranges between $132-148 \mu \mathrm{g} / \mathrm{mL}$ with HEX having the least $\mathrm{IC}_{50}$ value $132.64 \pm 1.17 \mu \mathrm{g} / \mathrm{mL}$ (Table 2). The fractions expressed different inhibitory effects on glucose uptake by yeast. At the concentration rang of $200-1000 \mu \mathrm{g} / \mathrm{mL}, \mathrm{AQE}, \mathrm{EAE}, \mathrm{HEX}$ and DEE expressed $18.96 \pm 6.68 \%, \quad 38.93 \pm 16.20 \%, \quad 36.16 \pm 7.41 \%$, and $21.59 \pm 2.43 \%$ inhibitory effects at glucose concentration of $20 \mathrm{mg} / \mathrm{mL}$ (Table 3) compared with $18.89 \pm 3.39 \%$ for reference drug metronidazole. Incubation of the fractions for 0,24 and 72 hours inhibited the formation of glycated haemoglobin at 2,10 and $20 \mathrm{mg} / \mathrm{mL}$ concentration of haemoglobin (Tables 4 - 6).

Table 1: In vitro $\alpha$-amylase inhibitory activities of Daucus carota seed extracts

\begin{tabular}{|c|c|c|c|c|c|c|c|c|c|}
\hline \multicolumn{10}{|c|}{$\alpha$-amylase activities } \\
\hline \multicolumn{2}{|c|}{ ACA } & \multicolumn{2}{|c|}{ AQE } & \multicolumn{2}{|c|}{ EAE } & \multicolumn{2}{|c|}{ HEX } & \multicolumn{2}{|c|}{ DEE } \\
\hline $\begin{array}{c}\text { Conc. } \\
(\mu \mathrm{g} / \mathrm{mL})\end{array}$ & $\%$ & $\begin{array}{c}\text { Conc. } \\
(\mu \mathrm{g} / \mathrm{mL})\end{array}$ & $\%$ & $\begin{array}{c}\text { Conc. } \\
(\mu \mathrm{g} / \mathrm{mL})\end{array}$ & $\%$ & $\begin{array}{c}\text { Conc. } \\
(\mu \mathrm{g} / \mathrm{mL})\end{array}$ & $\%$ & $\begin{array}{c}\text { Conc. } \\
(\mu \mathrm{g} / \mathrm{mL})\end{array}$ & $\%$ \\
\hline 2 & 18.53 & 200 & 36.25 & 200 & 31.45 & 200 & 12.18 & 200 & 12.26 \\
\hline 4 & 29.06 & 400 & 36.93 & 400 & 32.20 & 400 & 21.75 & 400 & 13.84 \\
\hline 6 & 38.46 & 600 & 41.74 & 600 & 37.20 & 600 & 25.53 & 600 & 20.96 \\
\hline 8 & 60.82 & 800 & 42.96 & 800 & 42.52 & 800 & 29.73 & 800 & 22.57 \\
\hline 10 & 68.66 & 1000 & 44.41 & 1000 & 45.18 & 1000 & 30.68 & 1000 & 24.00 \\
\hline \multicolumn{2}{|c|}{$\begin{array}{c}{ }^{*} \mathrm{IC}_{50}= \\
7.2 \pm 0.8^{\mathrm{a}}\end{array}$} & \multicolumn{2}{|c|}{$\begin{array}{c}{ }^{*} \mathrm{I}_{50}= \\
637.0 \pm 18.6^{\mathrm{b}}\end{array}$} & \multicolumn{2}{|c|}{$\begin{array}{c}{ }^{*} \mathrm{IC}_{50}= \\
603.0 \pm 25.8^{\mathrm{b}}\end{array}$} & \multicolumn{2}{|c|}{${ }^{*} \mathrm{IC}_{50}=$} & \multicolumn{2}{|c|}{$\begin{array}{l}{ }^{*} \mathrm{IC}_{50}= \\
>1000^{c}\end{array}$} \\
\hline
\end{tabular}

$\mathrm{ACA}=$ Acarbose, $\mathrm{AQE}=$ Aqueous extract, $\mathrm{EAE}=$ Ethyl acetate extract, $\mathrm{HEX}=\mathrm{n}$-hexane extract, $\mathrm{DEE}=$ Diethyl ether extract, $\mathrm{IC}_{50}=$ Half maximal inhibitory concentration

*Values are mean \pm Standard Error of Mean (SEM, triplicated determination), Values with different superscripts are significantly different $(\mathrm{p}<0.05)$

Table 2: In vitro $\alpha$-glucosidase inhibitory activities of Daucus carota seed extracts $\alpha$-glucosidase activities

ACA

AQE

EAE

HEX

DEE

\begin{tabular}{|c|c|c|c|c|c|c|c|c|c|}
\hline $\begin{array}{c}\text { Conc. } \\
(\mu \mathrm{g} / \mathrm{mL})\end{array}$ & $\%$ & $\begin{array}{c}\text { Conc. } \\
(\mu \mathrm{g} / \mathrm{mL})\end{array}$ & $\%$ & $\begin{array}{c}\text { Conc. } \\
(\mu \mathrm{g} / \mathrm{mL})\end{array}$ & $\%$ & $\begin{array}{c}\text { Conc. } \\
(\mu \mathrm{g} / \mathrm{mL})\end{array}$ & $\%$ & $\begin{array}{c}\text { Conc. } \\
(\mu \mathrm{g} / \mathrm{mL})\end{array}$ & $\%$ \\
\hline 2 & 36.73 & 200 & 94.87 & 200 & 90.66 & 200 & 95.31 & 200 & 91.36 \\
\hline 4 & 46.53 & 400 & 95.00 & 400 & 90.79 & 400 & 95.35 & 400 & 92.32 \\
\hline 6 & 48.27 & 600 & 95.09 & 600 & 91.01 & 600 & 95.44 & 600 & 92.94 \\
\hline 8 & 55.88 & 800 & 95.61 & 800 & 91.01 & 800 & 95.66 & 800 & 93.16 \\
\hline 10 & 60.64 & 1000 & 96.36 & 1000 & 91.58 & 1000 & 95.83 & 1000 & 93.42 \\
\hline \multicolumn{2}{|c|}{$\begin{array}{c}* \mathrm{IC}_{50}= \\
5.42 \pm 0.20^{\mathrm{a}}\end{array}$} & $\begin{array}{r}* 1 \\
135.8 \\
\end{array}$ & $21^{\mathrm{b}}$ & \multicolumn{2}{|c|}{${ }^{*} \mathrm{IC}_{50}=$} & \multicolumn{2}{|c|}{${ }^{*} \mathrm{IC}_{50}=$} & \multicolumn{2}{|c|}{$\begin{array}{c}* \mathrm{IC}_{50}= \\
143.56 \pm 0.49 \mathrm{e}\end{array}$} \\
\hline
\end{tabular}

$\mathrm{ACA}=$ Acarbose, $\mathrm{AQE}=$ Aqueous extract, $\mathrm{EAE}=$ Ethyl acetate extract, $\mathrm{HEX}=\mathrm{n}$-hexane extract, DEE = Diethyl ether extract, $\mathrm{IC}_{50}=\mathrm{Half}$ maximal inhibitory concentration $*$ Values are mean \pm Standard Error of Mean (SEM, triplicated determination), Values with different superscripts are significantly different ( $\mathrm{p}<0.05$ ) 
Table 3: Glucose uptake by yeast

\begin{tabular}{|c|c|c|c|c|c|c|}
\hline \multicolumn{7}{|c|}{ Glucose uptake (\%) } \\
\hline $\begin{array}{c}\text { Glucose } \\
\text { concentration }\end{array}$ & $2 \mathrm{mg} / \mathrm{mL}$ & $10 \mathrm{mg} / \mathrm{mL}$ & $20 \mathrm{mg} / \mathrm{mL}$ & $2 \mathrm{mg} / \mathrm{mL}$ & $10 \mathrm{mg} / \mathrm{mL}$ & $20 \mathrm{mg} / \mathrm{mL}$ \\
\hline $\begin{array}{l}\text { Fractions } \\
(\mu \mathrm{g} / \mathrm{mL})\end{array}$ & \multicolumn{3}{|c|}{$\mathrm{AQE}$} & \multicolumn{3}{|c|}{ EAE } \\
\hline 200 & $45.82 \pm 4.12^{\mathrm{a}}$ & $46.76 \pm 7.09^{a}$ & $52.97 \pm 2.23^{\mathrm{a}}$ & $44.33 \pm 2.50^{\mathrm{a}}$ & $47.10 \pm 6.75^{\mathrm{a}}$ & $12.55 \pm 3.64^{\mathrm{a}}$ \\
\hline 400 & $28.88 \pm 0.54^{a}$ & $27.67 \pm 8.77^{a}$ & $31.58 \pm 3.37 \mathrm{ab}$ & $42.78 \pm 0.67^{a}$ & $41.23 \pm 11.54^{\mathrm{a}}$ & $30.63 \pm 13.36^{b}$ \\
\hline 600 & $55.20 \pm 13.23^{\mathrm{a}}$ & $30.90 \pm 2.83^{\mathrm{a}}$ & $24.70 \pm 8.77^{b}$ & $45.14 \pm 1.15^{\mathrm{a}}$ & $45.75 \pm 3.64^{a}$ & $38.73 \pm 17.72^{\mathrm{b}}$ \\
\hline 800 & $46.63 \pm 16.40^{\mathrm{a}}$ & $34.21 \pm 1.96^{\mathrm{a}}$ & $10.12 \pm 7.42^{b}$ & $47.77 \pm 17.54^{\mathrm{a}}$ & $44.33 \pm 2.50^{\mathrm{a}}$ & $30.90 \pm 2.70^{\mathrm{b}}$ \\
\hline 1000 & $41.03 \pm 4.72^{\mathrm{a}}$ & $31.04 \pm 17.68^{\mathrm{a}}$ & $18.96 \pm 6.68^{\mathrm{b}}$ & $46.56 \pm 13.09^{a}$ & $48.79 \pm 4.12^{\mathrm{a}}$ & $38.93 \pm 16.20^{\mathrm{b}}$ \\
\hline Fractions $(\mu \mathrm{g} / \mathrm{mL})$ & \multicolumn{3}{|c|}{ HEX } & \multicolumn{3}{|c|}{ DEE } \\
\hline 200 & $56.00 \pm 5.39^{a}$ & $41.83 \pm 5.58^{\mathrm{a}}$ & $28.20 \pm 5.28^{\mathrm{a}}$ & $47.36 \pm 8.42^{\mathrm{a}}$ & $46.28 \pm 8.99 \mathrm{a}$ & $19.04 \pm 8.35^{\mathrm{a}}$ \\
\hline 400 & $14.70 \pm 9.85^{b}$ & $28.47 \pm 5.34^{\mathrm{a}}$ & $24.29 \pm 4.98^{\mathrm{a}}$ & $28.20 \pm 5.28^{\mathrm{b}}$ & $17.54 \pm 3.60^{c}$ & $36.43 \pm 7.47^{a}$ \\
\hline 600 & $28.34 \pm 8.10^{\mathrm{b}}$ & $57.75 \pm 9.84^{b}$ & $17.13 \pm 9.01^{\mathrm{a}}$ & $17.27 \pm 3.54^{\mathrm{c}}$ & $25.50 \pm 6.73^{c}$ & $61.53 \pm 8.62^{b}$ \\
\hline 800 & $17.13 \pm 9.01^{\mathrm{b}}$ & $33.60 \pm 3.39^{a}$ & $41.16 \pm 5.94^{b}$ & $18.21 \pm 4.75^{c}$ & $46.82 \pm 8.10^{\mathrm{a}}$ & $33.87 \pm 3.44^{\mathrm{a}}$ \\
\hline 1000 & $33.46 \pm 8.86^{b}$ & $14.84 \pm 4.84^{c}$ & $36.16 \pm 7.41^{\mathrm{a}}$ & $40.75 \pm 5.35^{\mathrm{a}}$ & $19.44 \pm 6.94^{c}$ & $21.59 \pm 2.43^{\mathrm{a}}$ \\
\hline Standard drug & \multicolumn{3}{|c|}{ Metronidazole } & & & \\
\hline $1000 \mu \mathrm{g} / \mathrm{mL}$ & $14.84 \pm 4.80^{\mathrm{a}}$ & $28.21 \pm 5.13^{\mathrm{b}}$ & $18.89 \pm 3.39^{\mathrm{a}}$ & & & \\
\hline
\end{tabular}

Table 4: Inhibition of haemoglobin glycosylation at zero hour of incubation

\begin{tabular}{|c|c|c|c|c|c|c|}
\hline \multirow[b]{2}{*}{$\begin{array}{l}\text { Glucose } \\
\text { concentration } \\
\text { Fractions } \\
(\mu \mathrm{g} / \mathrm{mL}) \\
\end{array}$} & \multicolumn{5}{|c|}{ Inhibition of haemoglobin glycosylation (\%, 0 hour) } & \multirow[b]{2}{*}{$20 \mathrm{mg} / \mathrm{mL}$} \\
\hline & \multicolumn{3}{|l|}{$\mathrm{AQE}$} & $\begin{array}{l}\mathbf{2 ~ m g / m L} \\
\mathrm{EAE}\end{array}$ & $10 \mathrm{mg} / \mathrm{mL}$ & \\
\hline 200 & $28.27 \pm 1.07^{a}$ & $36.94 \pm 4.18^{a}$ & $36.94 \pm 12.31^{\mathrm{a}}$ & $34.48 \pm 1.28^{\mathrm{a}}$ & $12.42 \pm 6.21^{\mathrm{a}}$ & $24.73 \pm 18.31^{a}$ \\
\hline 400 & $22.38 \pm 10.60^{\mathrm{a}}$ & $23.45 \pm 2.68^{b}$ & $24.20 \pm 3.64^{\mathrm{a}}$ & $34.48 \pm 7.28^{\mathrm{a}}$ & $37.90 \pm 3.43^{\mathrm{b}}$ & $30.30 \pm 11.67^{a}$ \\
\hline 600 & $33.08 \pm 2.46^{\mathrm{a}}$ & $31.37 \pm 2.68^{\mathrm{a}}$ & $10.92 \pm 1.21^{\mathrm{b}}$ & $32.76 \pm 3.85^{\mathrm{a}}$ & $29.44 \pm 3.75^{a}$ & $11.03 \pm 4.18^{\mathrm{b}}$ \\
\hline 800 & $23.66 \pm 11.24^{\mathrm{a}}$ & $25.70 \pm 2.78^{\mathrm{b}}$ & $20.66 \pm 7.60^{\mathrm{a}}$ & $38.54 \pm 4.71^{\mathrm{a}}$ & $18.52 \pm 2.03^{\mathrm{a}}$ & $28.91 \pm 2.36^{\mathrm{a}}$ \\
\hline 1000 & $26.23 \pm 18.31^{\mathrm{a}}$ & $17.02 \pm 12.31^{b}$ & $26.23 \pm 4.39^{a}$ & $24.20 \pm 11.56^{\mathrm{a}}$ & $22.81 \pm 3.10^{\mathrm{a}}$ & $15.20 \pm 7.71^{b}$ \\
\hline $\begin{array}{l}\text { Fractions } \\
(\mu \mathrm{g} / \mathrm{mL})\end{array}$ & \multicolumn{3}{|l|}{ HEX } & \multicolumn{3}{|l|}{ DEE } \\
\hline 200 & $47.86 \pm 4.54^{\mathrm{a}}$ & $49.79 \pm 3.32^{\mathrm{a}}$ & $48.93 \pm 1.18^{\mathrm{a}}$ & $28.69 \pm 14.99^{a}$ & $9.64 \pm 1.93^{\mathrm{a}}$ & $20.24 \pm 4.82^{\mathrm{a}}$ \\
\hline 400 & $47.43 \pm 5.03^{\mathrm{a}}$ & $25.80 \pm 15.10^{\mathrm{a}}$ & $27.30 \pm 17.67^{a}$ & $31.37 \pm 15.52^{\mathrm{a}}$ & $31.66 \pm 20.80^{\mathrm{b}}$ & $7.28 \pm 0.43^{b}$ \\
\hline 600 & $33.62 \pm 1.71^{\mathrm{b}}$ & $43.15 \pm 1.18^{\mathrm{a}}$ & $37.90 \pm 16.70^{\mathrm{a}}$ & $20.56 \pm 6.42^{\mathrm{a}}$ & $16.57 \pm 2.86^{\mathrm{c}}$ & $13.67 \pm 0.89 c$ \\
\hline 800 & $51.93 \pm 4.75^{\mathrm{a}}$ & $44.11 \pm 3.00^{\mathrm{a}}$ & $37.26 \pm 3.43^{\mathrm{a}}$ & $10.81 \pm 6.96^{b}$ & $22.06 \pm 11.78^{b}$ & $18.60 \pm 1.53^{c}$ \\
\hline 1000 & $37.15 \pm 2.46^{b}$ & $32.12 \pm 7.92^{\mathrm{b}}$ & $26.12 \pm 9.42^{a}$ & $10.17 \pm 3.53^{b}$ & $26.87 \pm 2.25^{b}$ & $21.09 \pm 2.89^{a}$ \\
\hline Standard drug & Gentamycin & & & & & \\
\hline $30 \mu \mathrm{g} / \mathrm{mL}$ & $6.42 \pm 1.83^{a}$ & $6.20 \pm 1.95^{\mathrm{a}}$ & $6.63 \pm 1.16^{\mathrm{a}}$ & & & \\
\hline
\end{tabular}


Table 5: Inhibition of haemoglobin glycosylation at twenty-four hours of incubation

\begin{tabular}{|c|c|c|c|c|c|c|}
\hline \multicolumn{7}{|c|}{ Inhibition of haemoglobin glycosylation (\%, 24 hours) } \\
\hline $\begin{array}{l}\text { Glucose } \\
\text { concentration }\end{array}$ & $2 \mathrm{mg} / \mathrm{mL}$ & $10 \mathrm{mg} / \mathrm{mL}$ & $20 \mathrm{mg} / \mathrm{mL}$ & $2 \mathrm{mg} / \mathrm{mL}$ & $10 \mathrm{mg} / \mathrm{mL}$ & $20 \mathrm{mg} / \mathrm{mL}$ \\
\hline $\begin{array}{l}\text { Fractions } \\
(\mu \mathrm{g} / \mathrm{mL})\end{array}$ & & $\mathrm{AQE}$ & & & EAE & \\
\hline 200 & $12.39 \pm 7.87 \mathrm{a}$ & $12.82 \pm 1.19^{a}$ & $16.06 \pm 1.11^{\mathrm{a}}$ & $22.74 \pm 3.56^{\mathrm{a}}$ & $12.07 \pm 3.66^{\mathrm{a}}$ & $17.89 \pm 1.22^{\mathrm{a}}$ \\
\hline 400 & $17.35 \pm 5.50^{\mathrm{a}}$ & $26.29 \pm 4.96^{b}$ & $10.67 \pm 2.05^{\mathrm{a}}$ & $7.00 \pm 4.20^{\mathrm{b}}$ & $15.84 \pm 4.6^{\mathrm{a}}$ & $9.81 \pm 7.22^{\mathrm{a}}$ \\
\hline 600 & $23.28 \pm 8.41^{\mathrm{a}}$ & $10.78 \pm 8.84^{\mathrm{a}}$ & $25.65 \pm 2.37^{a}$ & $14.22 \pm 1.72^{\mathrm{c}}$ & $18.64 \pm 3.99^{a}$ & $21.34 \pm 4.09^{\mathrm{b}}$ \\
\hline 800 & $1.08 \pm 0.43^{\mathrm{b}}$ & $3.13 \pm 2.48^{c}$ & $19.07 \pm 10.45^{\mathrm{a}}$ & $13.15 \pm 7.97 \mathrm{c}$ & $32.05 \pm 4.32^{b}$ & $16.81 \pm 9.05^{\mathrm{a}}$ \\
\hline 1000 & $2.05 \pm 1.62^{\mathrm{b}}$ & $15.84 \pm 13.25^{\mathrm{ab}}$ & $10.88 \pm 1.54^{\mathrm{a}}$ & $15.84 \pm 10.88^{\mathrm{a}}$ & $15.09 \pm 7.97^{a}$ & $5.82 \pm 5.17^{\mathrm{c}}$ \\
\hline $\begin{array}{l}\text { Fractions } \\
(\mu \mathrm{g} / \mathrm{mL})\end{array}$ & HEX & & & DEE & & \\
\hline 200 & $15.59 \pm 3.38^{\mathrm{a}}$ & $11.42 \pm 0.43^{a}$ & $20.91 \pm 12.50^{a}$ & $28.88 \pm 15.73^{a}$ & $10.34 \pm 1.94^{a}$ & $20.37 \pm 4.20^{\mathrm{a}}$ \\
\hline 400 & $19.40 \pm 0.22^{\mathrm{a}}$ & $26.72 \pm 13.15^{\mathrm{a}}$ & $32.44 \pm 5.19^{a}$ & $31.57 \pm 16.27^{a}$ & $28.34 \pm 3.81^{b}$ & $6.68 \pm 0.43^{b}$ \\
\hline 600 & $17.24 \pm 6.90^{\mathrm{a}}$ & $13.58 \pm 8.84^{a}$ & $6.36 \pm 1.19^{b}$ & $15.41 \pm 1.83^{b}$ & $8.62 \pm 5.82^{\mathrm{c}}$ & $8.73 \pm 5.28^{\mathrm{b}}$ \\
\hline 800 & $10.02 \pm 3.34^{\mathrm{a}}$ & $5.39 \pm 1.29^{\mathrm{b}}$ & $12.72 \pm 2.80^{c}$ & $10.24 \pm 7.00^{\mathrm{b}}$ & $21.55 \pm 11.85^{\mathrm{a}}$ & $14.33 \pm 6.57^{\mathrm{a}}$ \\
\hline 1000 & $18.21 \pm 11.31^{\mathrm{a}}$ & $14.66 \pm 11.42^{\mathrm{a}}$ & $10.78 \pm 1.94^{c}$ & $9.59 \pm 3.56^{c}$ & $27.69 \pm 2.26^{b}$ & $21.23 \pm 2.26^{\mathrm{a}}$ \\
\hline Standard drug & Gentamycin & & & & & \\
\hline $30 \mu \mathrm{g} / \mathrm{mL}$ & $23.92 \pm 2.41^{\mathrm{a}}$ & $14.95 \pm 6.97^{a}$ & $13.14 \pm 6.55^{\mathrm{a}}$ & & & \\
\hline
\end{tabular}

Table 6: Inhibition of haemoglobin glycosylation at seventy-two hours of incubation

\begin{tabular}{|c|c|c|c|c|c|c|}
\hline \multirow[b]{2}{*}{$\begin{array}{l}\text { Glucose } \\
\text { concentration } \\
\text { Fractions } \\
(\mu \mathrm{g} / \mathrm{mL})\end{array}$} & \multicolumn{5}{|c|}{ Inhibition of haemoglobin glycosylation (\%, 72 hours) } & \multirow[b]{2}{*}{$20 \mathrm{mg} / \mathrm{mL}$} \\
\hline & \multicolumn{3}{|l|}{ AQE } & \multicolumn{2}{|l|}{ EAE } & \\
\hline 200 & $15.06 \pm 2.13^{\mathrm{a}}$ & $18.32 \pm 4.04^{a}$ & $19.57 \pm 8.78^{a}$ & $42.91 \pm 7.94^{\mathrm{a}}$ & $13.66 \pm 2.73^{a}$ & $18.24 \pm 4.10^{\text {a }}$ \\
\hline 400 & $12.74 \pm 5.71^{a}$ & $11.48 \pm 3.46^{a}$ & $12.30 \pm 4.39^{a}$ & $22.96 \pm 5.90^{\mathrm{b}}$ & $9.52 \pm 2.49^{b}$ & $17.75 \pm 7.09^{\mathrm{a}}$ \\
\hline 600 & $17.19 \pm 2.92^{\mathrm{a}}$ & $7.59 \pm 2.33^{b}$ & $15.37 \pm 6.59^{a}$ & $9.41 \pm 7.65^{\mathrm{c}}$ & $15.43 \pm 6.65^{\mathrm{a}}$ & $16.04 \pm 3.16^{\mathrm{a}}$ \\
\hline 800 & $18.57 \pm 7.65^{\mathrm{a}}$ & $33.75 \pm 6.40^{c}$ & $34.19 \pm 8.77 \mathrm{~b}$ & $7.38 \pm 4.50^{\mathrm{c}}$ & $16.29 \pm 4.03^{\mathrm{a}}$ & $14.81 \pm 6.90^{\mathrm{a}}$ \\
\hline 1000 & $39.21 \pm 2.57^{b}$ & $39.52 \pm 7.82^{c}$ & $28.04 \pm 7.51^{\mathrm{a}}$ & $9.16 \pm 1.25^{\mathrm{c}}$ & $13.68 \pm 7.90^{\mathrm{a}}$ & $26.27 \pm 7.64^{\mathrm{a}}$ \\
\hline $\begin{array}{l}\text { Fractions } \\
(\mu \mathrm{g} / \mathrm{mL})\end{array}$ & \multicolumn{3}{|l|}{ HEX } & \multicolumn{3}{|l|}{ DEE } \\
\hline 200 & $36.57 \pm 3.83^{a}$ & $6.46 \pm 1.69^{a}$ & $22.88 \pm 6.61^{a}$ & $35.26 \pm 12.08^{a}$ & $31.22 \pm 14.57 \mathrm{a}$ & $27.42 \pm 2.69^{\mathrm{a}}$ \\
\hline 400 & $25.22 \pm 8.44^{\mathrm{a}}$ & $43.48 \pm 7.67^{b}$ & $36.20 \pm 8.64 b$ & $18.32 \pm 6.27^{b}$ & $26.16 \pm 12.99$ & $13.80 \pm 2.13^{b}$ \\
\hline 600 & $30.99 \pm 7.30^{\mathrm{a}}$ & $17.86 \pm 1.93^{a}$ & $17.74 \pm 3.85^{a}$ & $18.32 \pm 3.92^{b}$ & $15.43 \pm 11.67^{\mathrm{b}}$ & $3.26 \pm 0.63^{c}$ \\
\hline 800 & $22.08 \pm 2.51^{\mathrm{a}}$ & $5.27 \pm 1.75^{\mathrm{a}}$ & $43.16 \pm 5.25^{b}$ & $22.33 \pm 3.05^{b}$ & $5.90 \pm 3.26^{c}$ & $11.52 \pm 1.49^{b}$ \\
\hline 1000 & $23.88 \pm 6.98^{a}$ & $16.44 \pm 2.25^{\mathrm{a}}$ & $28.11 \pm 8.71^{\mathrm{b}}$ & $11.42 \pm 1.51^{\mathrm{b}}$ & $45.80 \pm 6.02^{\mathrm{a}}$ & $12.34 \pm 1.55^{\mathrm{b}}$ \\
\hline Standard drug & Gentamycin & & & & & \\
\hline $30 \mu \mathrm{g} / \mathrm{mL}$ & $12.04 \pm 1.75^{a}$ & $24.21 \pm 3.81^{\mathrm{b}}$ & $21.70 \pm 2.64^{\mathrm{b}}$ & & & \\
\hline
\end{tabular}

\subsection{Discussion}

Diabetes mellitus (DM) are among the common disorders of the endocrine system resulting in metabolic disorders and which further leads to multiple organ damage syndrome. The inhibition of key enzymes such as $\alpha$-amylase and $\alpha$-glucosidase which are involved in the digestion of carbohydrates can significantly lower observed

This journal is (C) The Nigerian Young Academy 2021 postprandial increases of blood glucose after a mixed diet. The inhibition of these key enzymes are important strategy in the management of the level of postprandial blood glucose in type 2 diabetic patients and among borderline patients (Ali et al., 2006). $\alpha$ amylase inhibitors including voglibose, miglitol and acarbose (a reference drug use in the present study) are among clinical drugs use as treatment, but their prices are high and clinical side effects are reported (Khoo, 2017). More so, intestinal $\alpha$-glucosidases are key

Annals of Science and Technology 2021 Vol. 6(1) 26-33 |30 
enzyme for carbohydrate digestion that are located at the epithelium of the small intestine. $\alpha$-glucosidase are key enzymes recognized as a therapeutic target for the modulation of postprandial hyperglycemia, the initial metabolic abnormality observed in Type 2 DM (Scott et al., 2000).

The in vitro $\alpha$-amylase and $\alpha$-glucosidase inhibitory studies demonstrated that Daucus carota extract has both $\alpha$-glucosidase and $\alpha$-amylase inhibitory activities. The percentage inhibition at 200,400 , 600,800 and $1000 \mu \mathrm{g} / \mathrm{mL}$ concentrations of plant extract showed a concentration dependent percentage inhibition (Table 1 and Table 2). $\alpha$ - amylase inhibitors are also called starch blockers since they prevent or slow down the absorption of starch mainly by blocking the hydrolysis of 1, 4-glycosidic linkages of starch during digestion and other oligosaccharides into maltriose, maltose and other simple sugars (Wadkar et al., 2008). In the study, the ethyl acetate (EAE) and nhexane (HEX) extract expressed higher $\alpha$ - amylase inhibitory and $\alpha$ glucosidase inhibitory activities. In the study acarbose was used as the positive control, it inhibited the $\alpha$-amylase activity and $\alpha$ - glucosidase inhibitory activities better when compared with the extracts. The results obtained from the study indicated that the ethyl acetate and nhexane fractions are also potent $\alpha$-amylase and $\alpha$ - glucosidase inhibitions.

Glycosylated haemoglobin (glycohaemoglobins) are formed when a ketoamine reaction occurs between the N-terminal amino acid of the $\beta$ chain of haemoglobin and glucose. Glycohaemoglobins level is important indicators for long-term blood glucose control (McCowen and Smith, 2005). The results suggest that the plant extract inhibits the binding of glucose to haemoglobin with evidence from the glycated haemoglobin assay (Table 4 -6). Advance glycation end product (AGEs) is a known source of free radicals in diabetes, and their accumulation aggravates the state of an increased oxidative stress in DM (Adisa et al., 2004). Thus, hindering the formation of AGEs implies decreased levels of free radicals in diabetes, and could reduce further diabetic complication. In management of diabetic mellitus, reducing properties of monosaccharides are important. More so, direct glycosylation reactions of monosaccharide lead to enolize, reduce molecular oxygen and yielding hydrogen peroxide, $\alpha$-ketoaldehydes and free radical intermediates (Wolff and Dean, 1987). The occurrence of this process in vivo, contributes significantly to the elevated plasma peroxides levels in diabetics complication and may contribute to protein modification reactions perform with glucose in vitro.

The mechanism of glucose transport across the cell membrane of yeast has received attention, and are utilized as in vitro screening methods for various compounds/medicinal plants to access their hypoglycaemic potentials (Maier et al., 2002). The results of the present study revealed that Daucus carota extract decrease glucose uptake in yeast cells at various glucose concentrations of 5, 10 and 20 $\mathrm{mg} / \mathrm{mL}$. This was measured by the amount of glucose remaining in the medium after a specific time which serve as an indicator of glucose uptake by the yeast cells (Ahmed and Urooj, 2010). Recent studies by Teusink et al. (1998) on the means of transport of non-metabolizable sugars and glycosides across yeast cell membrane indicates that the sugars are transported across membranes through stereospecific membrane carriers. Saccharomyces cerevisiae glucose transport are extremely complex and generally, it is suggested that glucose are transported across yeast cells by a facilitated diffusion process (Teusink et al., 1998). Facilitated carriers process are specific types of process that transport solutes (glucose) down concentration gradient. The effective transport is only attained when there is effective removal of intracellular glucose (Teusink et al., 1998). Thus, glucose transport will occur only if the intracellular glucose is effectively reduced by the means of their utilization for metabolic processes. The present study, suggests that Daucus carota seed extract is capable of inhibiting glucose uptake, which in turn suggests that it can regulate glucose uptake through the control of blood glucose levels as also suggested by Ahmed et al. (2009).

Medicinal plants and their products usually contain phytochemicals which includes phenolic, flavonoids, terpenoids, coumarins and some other constituents, which help in controlling blood glucose levels. Thereby, the serve as alternative medicines to treat diabetes due to the presences of relevance chemical composition. AQE and DEE of Daucus carota contains flavonoids, alkaloids, saponins, steroids, tannins and phenols (Tijjani et al., 2019). The maintenance of plasma glucose levels for an extended period of time under a variety of dietary conditions is an important and closely regulated processes (Nair et al., 2013). Presence of fibers in diet, along with some phytochemicals are important in combating diabetes disorders, through several proposed mechanisms which could include enhancing insulin releasing activity, manipulation of glucose transporters, inhibition of carbohydrate metabolizing enzymes and $\beta$-cell regeneration (Jenkins et al. 1978; Lopez et al. 1996; Sairam and Urooj, 2013).

\section{Conclusion}

The results show that fractions from aqueous seed extract of Daucus carota possess in vitro andiabetic potentials with ethyl acetate (EAE) and n-hexane (HEX) fractions having most promising inhibitory activities against $\alpha$-amylase and $\alpha$-glucosidase respectively. The fractions decreased \% glucose uptake by yeast, however, not in the same manner with metronidazole and prevented haemoglobin glycosylation which were however, lower in most cases when compared with gentamicin. Further studies may ascertain the observed preventive haemoglobin glycosylation activities in vivo.

\section{Declaration of Conflict of Interests}

The authors declare no conflict of interests.

\author{
Authors' Contributions \\ Conception: [HT] \\ Design: [HT] \\ Execution: [SAI] \\ Interpretation: [HT] \\ Writing the paper: [HT, SAI]
}

\section{References}

Adisa, R. A, Oke, J, Olomu, S. A., Olorunsogo, O., 2004, Inhibition of human haemoglobin glycosylation by flavonoid containing leaf extract of Cnestis ferruginea. Journal of the Cameroon Academy of Sciences, 4: 
351-359. https://africaneditors.org/journal/JCAS/abstract/37623$\underline{79307}$

Ahmed, F., Sairam, S., Urooj, A., 2009, Effect of various Ayurvedic formulations and medicinal plants on carbohydrate hydrolyzing enzymes and glucose uptake by yeast cells-an in vitro study. J Pharm Res., 2:563-8.

Ahmed, F., Urooj, A., 2010, In vitro studies on the hypoglycemic potential of Ficus racemosa stem bark. J Sci Food Agric., 90:397-401. DOI: $10.1002 /$ isfa.3828

Ali, H., Houghton, P.J., Soumyanath, A., 2006, $\alpha$-Amylase inhibitory activity of some Malaysian plants used to treat diabetes; with particular reference to Phyllanthus amarus. J Ethnopharmacol, 107: 449-455. DOI: $10.1016 /$ i.jep.2006.04.004

Andrade-Cetto, A., Becerra-Jimennez, J., Cardenes, R., 2008. Alpha glucosidase inhibiting activity of some Mexican plants used in treatment of type 2 diabetes mellitus. Journal of ethnopharmacology, 116: 27-32. DOI: $10.1016 /$ j.jep.2007.10.031

Cirillo, V.P., 1962, Mechanism of glucose transport across the yeast cell membrane. J Bacteriol, 84: 485-91. doi:10.1128/JB.84.3.

International Diabetes Federation, (IDF) Diabetes atlas. 8th ed. Brussels: International Diabetes Federation; 2019.

Jenkins, D.J.A., Wolever, T.M.S., Leeds, A.R., Gassul, M.A., Haisman, P., Dilawari, J., Goff, D.V., Metz, G.L., Alberti, K.G.M.M., 1978, Dietary fibers, fiber analogues and glucose tolerance: importance of viscosity. Brit Med J, 1:1392-1394. doi: 10.1136/bmj.1.6124.1392

Khaki, A., 2011, Effect of Ethanolic Extract of Carrot seed on Blood Glucose level and Liver Tissue in Streptozotocin-Induced Diabetic Rat. Medical Journal of Tabriz University of Medical Science, 33:3.

Khoo, C. M., 2017, Diabetes Mellitus Treatment, Editor(s): Stella R. Quah, International Encyclopedia of Public Health (Second Edition), Academic Press, 288-293.

Kuppusamy, A., Muthusamy, U., Swashanmugum, T., Subhadradevi, V., Kalyanasubramaniam, R., Sambathkumar, R., 2011, In vitro ( $\alpha$ Glucosidase and $\alpha$-Amylase) inhibition and in vivo antidiabetic property of Phytic acid (ip 6 ) in Streptozotocin-Nicotinamide-induced type 2 Diabetes mellitus (NIDDM) in rats. Journal of complementary and integrative medicine, 8: 1553-3840. dOI: 10.2202/1553$\underline{3840.1483}$

Lopez, G., Ros, G., Rincon, F., Periago, M.J., Martinez, M.C., Ortuno, J., 1996, Relationship between physical and hydration properties of soluble and insoluble fiber of artichoke. J Agric Food Chem, 44:27732778. https://doi.org/10.1021/if9507699

Maier, A., Völker, B., Boles, E., Fuhrmann, G.F., 2002, Characterisation of glucose transport in Saccharomyces cerevisiae with plasma membrane vesicles (countertransport) and intact cells (initial uptake) with single Hxt1, Hxt2, Hxt3, Hxt4, Hxt6, Hxt7 or Gal2 transporters. FEMS Yeast Res., 2(4): 539-50. DOI: 10.1111/j.15671364.2002.tb00121.x
McCowen, K.C., Smith, R.J., 2005, Diabetes Mellitus Classification and Chemical Pathology, Editor(s): Benjamin Caballero, Encyclopedia of Human Nutrition (Second Edition), Elsevier, 543-551.

Nair, S. S., Kavrekar, V., Mishra, A., 2013, Evaluation of in vitro antidiabetic activity of selected plant extracts. Int J Pharm Pharm Sci Invent., 2:12-9.

Olatunde, A., Tijjani, H., Ishola, A.A., Egbuna, C., Hassan, S., Akram, M., 2020, Carotenoids as Functional Bioactive Compounds. In: Egbuna C., DableTupas G. (eds) Functional Foods and Nutraceuticals. Springer, Cham. Pp 415-444. https://doi.org/10.1007/978-3-030-42319-3 20

Pouraboli, I., Ranjbar, B., 2015, The effect of Daucus carota seeds extract on lipid profile, LFT and kidney function indicators in streptozocin-induced diabetic rats. Int J Plant Sci Ecol. 3:84-7.

Sairam, S., Urooj, A.. 2013, Artocarpusaltilis-mode of anti hyperglycemic activity: elucidation by suitable in-vitro and exvivo techniques. Int J Pharm Sci Res., 4:3013.

DOI: http://dx.doi.org/10.13040/IJPSR.0975-8232.4(8).3013-19

Scott, L.J., Spencer, C.M., 2000, Miglitol: a review of its therapeutic potential in type 2 diabetes mellitus. Drugs, 59: 521-549. DOI: $10.2165 / 00003495-200059030-00012$

Singh, K., Singh, N., Chandy, A., Manigauha, A., 2012, In vivo antioxidant and hepatoprotective activity of methanolic extracts of Daucus carota seeds in experimental animals. Asian Pac J Trop Biomed., 2:385-8. DOI: 10.1016/S2221-1691(12)60061-6

Teusink, B., Diderich, J. A., Westerhoff, H. V., van Dam, K., Walsh, M. C., 1998, Intracellular glucose concentration in derepressed yeast cells consuming glucose is high enough to reduce the glucose transport rate by $50 \%$. Journal of bacteriology, 180(3): 556-562.

DOI: $10.1128 /$ JB.180.3.556-562.1998

Tijjani, H., Adegunloye, A.P., Uba, H., Joel, E.B., Olatunde, A., 2020b, Antioxidant activities of aqueous and ethyl acetate fractions of Daucus carota L. seed against triton X-100 induced oxidative stress in mice. Scientific African. 8, e00429.

https://doi.org/10.1016/j.sciaf.2020.e00429

Tijjani, H., Mohammed, A., Adegunloye, A.P., 2019, Phytochemicals and in vitro antioxidant studies of Daucus carota L. seed extracts, 5th International Electronic Conference on Medicinal Chemistry, 1-30. https://sciforum.net/paper/view/6278. DOI:10.3390/ECMC201906278

Tijjani, H., Mohammed, A., Ahmed, F., Yahaya, H., Zakka, N., 2020c, In vitro antioxidant activity-guided fractionation of Daucus carota $\mathrm{L}$. seed extract. Proceedings of the Nigerian Academy of Science, 13(2): 74 85. https://nasjournal.org.ng/index.php/pnas/article/view/279

Tijjani, H., Mohammed, A., Idris, Z.L., Adegunloye, P.A., Luka, C.D. and Alhassan, A.J., 2017, Current status of antidiabetic plants in the Lamiaceaefamily, OmniScriptin GmbH \& Co. KG Publisher, Germany, ISBN 198-3-330-86902-8, Pp 1-64.

Tijjani, H., Mohammed, A., Muktar, S., Musa, S., Abubakar, Y., Adegunloye, A. P., Ishola, A. A., Joel, E. B., Luka, C. D., Alhassan, A. J., 2020a, Antioxidant and antihyperlipidemic effects of aqueous seed 
extract of Daucus carota $\mathrm{L}$. in triton $\times 100$-induced hyperlipidemic mice. J App Biol Biotech., 8(1):76-83. DOI: 10.7324/JABB.2020.80113

Uloko, A. E., Musa, B. M., Ramalan, M. A., Gezawa, I. D., Puepet, F. H., Uloko, A. T., Borodo, M. M., Sada, K. B., 2018, Prevalence and Risk Factors for Diabetes Mellitus in Nigeria: A Systematic Review and Meta-Analysis. Diabetes therapy: research, treatment and education of diabetes and related disorders, 9(3):1307-1316.

doi: $\underline{10.1007 / \mathrm{s} 13300-018-0441-1}$

Uloko, A.E., Ofoegbu, E.N., Chinenye, S., Fasanmade, O.A., Fasanmade, A.A., Ogbera, A.O., Ogbu, O.O., Oli, J.M., Girei, B.A., Adamu, A., 2008, Profile of Nigerians with diabetes mellitus - Diabcare Nigeria study group (2008): Results of a multicenter study. Indian J Endocrinol Metab., 16(4): 558-64. doi: 10.4103/2230-8210.98011

Mani, V., Parle, M., Ramasamy, K., Abdul Majeed, A.B., 2010, Antidementia potential of Daucus carota seed extracts in rats. Pharmacologyonline, 1:552-65.

Wadkar, K.A., Magdum, C.S., Patil, S.S., Naikwade, N.S., 2008, Antidiabetic potential and indian medicinal plants. Journal of Herbal Medicine and Toxicology, 2(1): 45-50.
WHO, 2009, World Health Organization; African Region, Nigeria: Emerging epidemics. http://www.whoint/countries/nga/areas/hiv Len/index.html Accessed date 04/12/2019.

Wolff, S.P., Dean, R.T., 1987, Glucose auto-oxidation and protein modification. The potential role of 'autoxidative glycosylation' in diabetes. Biochem, 245: 243-250. doi: 10.1042/bj2450243 